\title{
Inflationsziel und Inflationsmessung in der Eurozone im Wandel
}

\author{
Viele Menschen im Euroraum haben zunehmend das Gefühl, dass der allgemeine \\ Kaufkraftverlust des Geldes sehr viel höher ist als die offiziell gemessenen Inflationsraten. So \\ lag Umfrageergebnissen zufolge z. B. im ersten Quartal 2021 die gefühlte Inflation im Euroraum \\ bei 4,5\%, während die offiziell gemessene Inflationsrate $1 \%$ betrug. Das könnte daran liegen, \\ dass Preissteigerungen subjektiv stärker wahrgenommen werden als Preissenkungen. Es kann \\ aber ebenso gut daran liegen, wie Preisstabilität gemessen wird und wie sich die Umsetzung \\ des Ziels der Preisstabilität durch die Europäische Zentralbank verändert hat.
}

Artikel 127 (1) des Vertrags über die Arbeitsweise der Europäischen Union (AEUV) gibt die Preisstabilität als vorrangiges Ziel des Europäischen Systems der Zentralbanken (ESZB) vor. Eine nähere Erläuterung hierzu findet sich allerdings nicht. Es bleibt jedoch festzuhalten, dass als Ziel Preisstabilität formuliert ist und nicht eine „optimale“ Inflationsrate. Im Oktober 1998 operationalisierte der EZB-Rat Preisstabilität als Anstieg des Harmonisierten Verbraucherpreisindex (HVPI) für das Euro-Währungsgebiet von (mittelfristig) unter $2 \%$ gegenüber dem Vorjahr (Europäische Zentralbank, 1999). Der Maximalwert von $2 \%$ sollte vor allem eine Sicherheitsmarge gegen das mögliche Risiko dauerhaft fallender Preise (Deflation) bilden, eventuellen systematischen Messfehlern der Preisstatistik Rechnung tragen und ausreichend Spielraum für strukturelle Inflationsdifferenzen im Euroraum lassen. ${ }^{1}$ Damit wurde im Ergebnis eine Art HVPI-Inflationskorridor zwischen $0 \%$ und $2 \%$ festgelegt (siehe hellblaue Fläche, Abbildung 1).

Um vorausschauend zukünftige Preisentwicklungen einschätzen zu können, legte die EZB 1999 zudem einen

(c) Der/die Autor:in(nen) 2021. Open Access: Dieser Artikel wird unter der Creative Commons Namensnennung 4.0 International Lizenz veröffentlicht (creativecommons.org/licenses/by/4.0/deed.de).

Open Access wird durch die ZBW - Leibniz-Informationszentrum Wirtschaft gefördert.

1 Vor diesem Hintergrund hat der EZB-Rat die folgende Definition beschlossen: „Preisstabilität wird definiert als Anstieg des Harmonisierten Verbraucherpreisindex (HVPI) für das Euro-Währungsgebiet von unter 2\% gegenüber dem Vorjahr." In Einklang mit dieser Definition „muss Preisstabilität mittelfristig beibehalten werden“. Die Formulierung „unter $2 \%$ “ gibt unzweideutig die Obergrenze für die am HVPI gemessene Inflationsrate an, die mit Preisstabilität vereinbar ist. Gleichzeitig macht die Verwendung des Wortes „Anstieg“ in der Definition klar, dass Deflation, d. h. anhaltende Rückgänge des HVPIIndex, nicht als mit Preisstabilität vereinbar angesehen würden. (Europäische Zentralbank, 1999, 51).
Referenzwert für das Geldmengenwachstum M3 in Höhe von 4,5\% (als erste Säule der damaligen geldpolitischen Strategie) fest. ${ }^{2}$ Darüber hinaus wurde eine breite Palette an Indikatoren wie Geschäftsklima, Löhne und Wechselkurse etc. bestimmt, die Hinweise auf die zukünftige Entwicklung der Inflation geben (zweite Säule). Die 2-SäulenStrategie der EZB galt als ein Kompromiss zwischen einer nicht zuletzt von der Deutschen Bundesbank befürworteten Geldmengenstrategie und einer Inflationszielstrategie, wie sie zunehmend in den USA und Großbritannien vertreten wurde.

In den Jahren 2001 bis 2007 lag die Veränderungsrate des HVPI in etwa an der $2 \%$-Obergrenze (vgl. Abbildung 1), während das Geldmengenwachstum M3 meist deutlich über dem Referenzwert von 4,5\% lag (vgl. Abbildung 2). Im Mai 2003 wendete man sich von der geldpolitischen Konzeption der Deutschen Bundesbank weiter ab, da „die

2 Die Ableitung des Referenzwerts für das Geldmengenwachstum M3 basierte konzeptionell auf der Quantitätsgleichung, die das nominale Sozialprodukt multiplikativ mit der Geldmenge und der Umlaufgeschwindigkeit des Geldes verknüpft. Danach ergibt sich bei einer gesamtwirtschaftlichen Preissteigerungsrate von (maximal) $2 \%$, einer prognostizierten mittelfristigen Wachstumsrate des realen BIP von $2 \%$ (p. a.) und einer geschätzten Abnahme der Trendrate der Umlaufgeschwindigkeit von $-0,5 \%$ (p. a.) der Referenzwert für das Wachstum der Geldmenge M3 von 4,5\% (p. a.).

Prof. Dr. Gunther Schnabl ist Leiter des Instituts für Wirtschaftspolitik der Universität Leipzig.

Tim Sepp ist dort wissenschaftlicher Mitarbeiter. 
Abbildung 1

Veränderungsraten des HVPI in der Eurozone

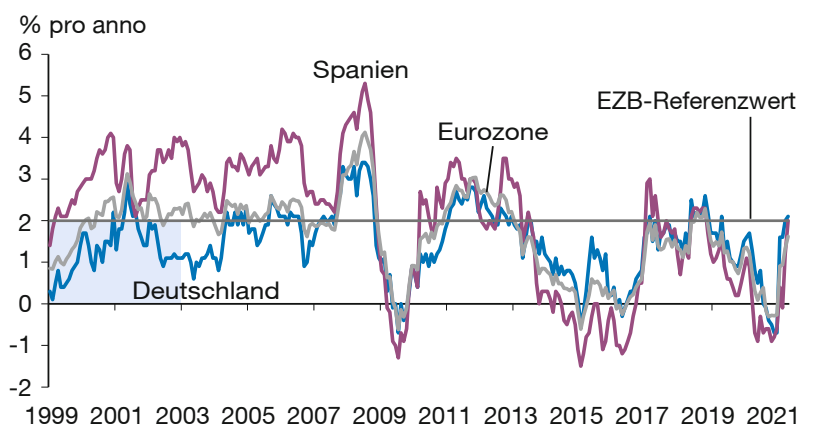

Anmerkung: Die hellblaue Fläche simuliert die Dauer des Inflationskorridors.

Quelle: EZB.

ständige Notwendigkeit, zu versuchen, den wachsenden monetären Überhang ohne entsprechende Inflation am Horizont wegzuerklären, zu einer peinlichen Übung wurde“ (Constancio, 2018). Der EZB-Rat strebte nunmehr ohne größeren Erklärungsaufwand als Zielwert einen Anstieg des HVPI von mittelfristig unter, aber nahe $2 \%$ gegenüber dem Vorjahr (Europäische Zentralbank, 2003) an. ${ }^{3}$ Der Referenzwert für das Geldmengenwachstum M3 wurde fallengelassen, und die erste - monetäre - Säule der Geldpolitik der EZB wurde zur zweiten Säule „degradiert“.

In der Folge lag bis 2007 das Geldmengenwachstum weit über dem ehemaligen Referenzwert (vgl. Abbildung 2), was sich vor allem in höheren Inflationsraten in der südlichen Eurozone und Irland kombiniert mit dort stark steigenden Immobilienpreisen widerspiegelte. Da die Inflation im Norden der Eurozone niedrig blieb (vgl. Abbildung 1) und die EZB ihre Geldpolitik auf den Durchschnitt der Eurozone ausrichtete (Prinzip des One Size Fits All), wirkte sie den Übertreibungen in der südlichen Eurozone nicht entgegen. Die Zinserhöhungen der EZB ab Dezember 2005 (vgl. Abbildung 3), die auch mit wachsenden Inflationsgefahren im Euroraum begründet wurden, brachten die Übertreibungen im südlichen Euroraum und Irland zum Ende.

Spätestens die 2010 einsetzende europäische Finanzund Schuldenkrise veränderte die Geldpolitik der EZB grundlegend. Die EZB senkte erneut die Leitzinsen auf

3 Im Gegensatz zum Verständnis des EZB-Rats 2003 wurde das Ergebnis der Strategieüberprüfung später als eine Strategieänderung hin zum kurzfristigen Inflation Targeting uminterpretiert. Die Absichtserklärung, mittelfristig eine Inflationsrate von unter, aber nahe $2 \%$ anzustreben, wurde zum selbst auferlegten kurzfristigen Punktziel von 1,9\%. Die EZB vollzog damit einen Paradigmenwechsel. Die „Zwei-Säulen-Strategie spielte nur noch formal eine Rolle“ (Stark, Mayer und Schnabl, 2020).
Abbildung 2

Veränderungsrate der Geldmenge M3

in der Eurozone

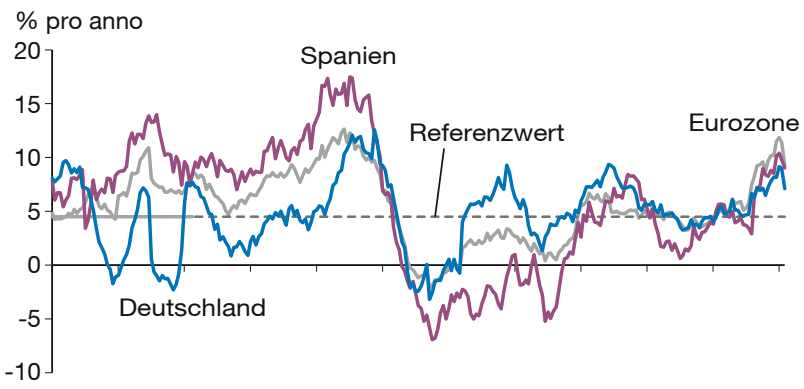

199920012003200520072009201120132015201720192021

Quelle: EZB.

nunmehr unter null (vgl. Abbildung 3) und weitete in mehreren Schritten insbesondere ab 2012 ihre Anleihekäufe stark aus. Bis zu diesem Zeitpunkt hatte sie keine Staatsund Unternehmensanleihen direkt in ihre Bilanz genommen, sondern die Zinsen überwiegend mit verschiedenen großvolumigen Refinanzierungsgeschäften mit (zum Teil sehr langen Laufzeiten) gesteuert.

Die umfangreichen Staatsanleihekäufe im Zuge von Rettungsaktionen ${ }^{4}$ für eigentlich insolvente Euro-Krisenstaaten (sowie flankierend eine expansiv ausgerichtete Forward Guidance) wurden damit gerechtfertigt, dass die Veränderungsraten des HVPI für den Euroraum deutlich unter dem neuen Nahe-2\%-Punktziel der EZB lagen. Bei Fortbestand des Korridors von $0 \%$ bis $2 \%$ wäre diese Rechtfertigung nicht möglich gewesen. Inzwischen liegt das Volumen der angekauften Staatsanleihen durch das Eurosystem bei ca. 3.500 Mrd. Euro. Zugleich stiegen die Bilanzsumme des Eurosystems und die geschaffene Zentralbankgeldmenge auf immer neue Höchststände.

Die auf die strukturell schwache wirtschaftliche Lage im südlichen Euroraum ausgerichtete expansive Geldpolitik der EZB wirkte in Deutschland prozyklisch und trug dort ab 2012 durch immer niedrigere Nominal- und Realzinsen zu stark steigenden Immobilienpreisen bei und begünstigte durch den Abwertungsdruck auf den Euro den Export. Die damit verbundene "Schönwetter-Konjunktur“ fand im März 2020 mit den Lockdown-Maßnahmen in ganz Europa ein abruptes Ende, nachdem sich die Konjunkturaussichten bereits 2019 eingetrübt hatten und

4 „Im Rahmen unseres Mandats ist die EZB bereit, alles zu tun, was nötig ist, um den Euro zu erhalten. Und glauben Sie mir, es wird genug sein" (Draghi, 2012). 
Abbildung 3

Leitzinsen der EZB

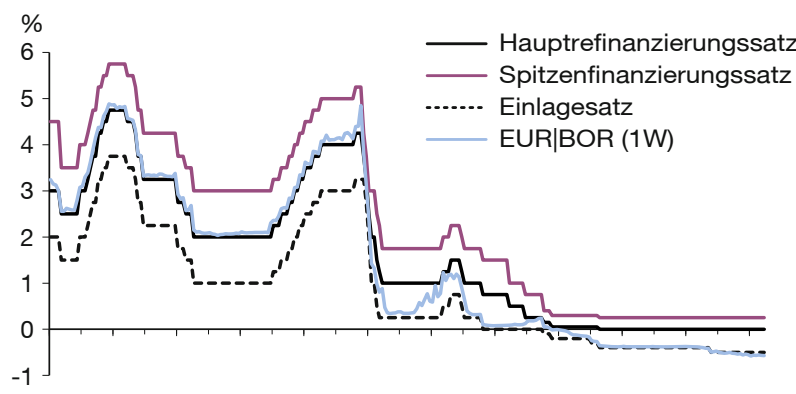

199920012003200520072009201120132015201720192021

Quelle: EZB.

die EZB ihre Anleihekäufe im Rahmen des Public Sector Purchase Programm bereits im November 2019 in Höhe von 20 Mrd. Euro pro Monat wieder aufgenommen hatte. Die EZB reagierte mit einem Pandemischen Notfallkaufprogramm (Umfang bis $1.850 \mathrm{Mrd}$. Euro), anhaltend ultraniedrigen Zinsen sowie der Ausweitung der längerfristigen gezielten Refinanzierungsgeschäfte mit einem negativen Zins von bis zu $-1 \%$ im Umfang bis zu 3.300 Mrd. Euro (Schnabl und Sonnenberg, 2020).

Für 2021 wurde eine Überprüfung der geldpolitischen Strategie angekündigt. Die EZB-Präsidentin Christine Lagarde hat sich für den Übergang zu einem symmetrischen Inflationsziel ausgesprochen. Dieses würde die Möglichkeit eröffnen, die $2 \%$-Marke zu überschreiten, um die niedrige Inflation der Vergangenheit „auszugleichen“ (Lagarde, 2020). Die Inflationsrate könnte für längere Zeit über $2 \%$ steigen, weil sie vor der Krise lange Zeit unter $2 \%$ gelegen hat (vgl. Abbildung 1). Zuletzt wurde vom Rat der EZB ein symmetrisches Punktziel von $2 \%$ beschlossen (EZB, 2021a). Dies würde es zulassen, dass die Inflation vorübergehend moderat über das Punktziel hinaus geht. Diese Ankündigung löste Erwartungen aus, dass die EZB die Inflationsrate zeitweise deutlich über $2 \%$ steigen lassen wird (Börsenzeitung, 2021). Dies entspricht de facto einem Wechsel zu einem Preisniveauziel ${ }^{5}$ anstelle des bisherigen Preisratenziels und einer Anhebung des bisherigen Inflationsziels, die in eine Phase des wachsenden Inflationsdrucks fällt.

Denn das Volumen der EZB-Bilanz ist nochmals auf zuletzt knapp 8.000 Mrd. Euro stark gewachsen (vgl. Ab-

5 Das Zielpreisniveau würde sich pro Jahr um $2 \%$ erhöhen, wobei der Startpunkt ungewiss ist.
Abbildung 4

Volumen der Bilanz des Eurosystems

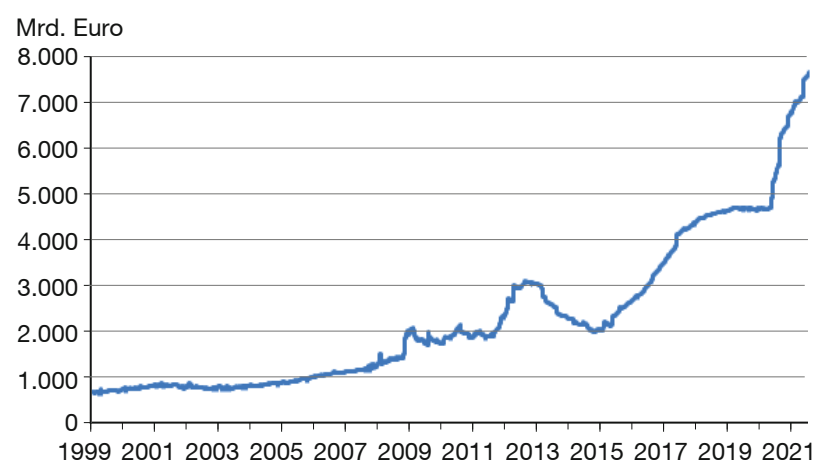

Quelle: EZB.

bildung 4), während das Volumen der produzierten Güter und Dienstleistungen 2020 gesunken ist. Die LockdownMaßnahmen gingen mit einer starken Ausgabenzurückhaltung der Haushalte einher, sodass die Einlagen bei den Banken und damit die Geldmenge M3 stark angestiegen sind (vgl. Abbildung 2). ${ }^{6}$ Die EZB-Direktorin Schnabel erwartet inzwischen für 2021 eine Inflationsrate von über $3 \%$. Sie betrachtet diese aber als kurzfristig und geldpolitisch für nicht relevant (ntv, 2021). Neueste Schätzungen der Deutschen Bundesbank gehen für 2021 von vorübergehend $4 \%$ Inflation für Deutschland aus (FAZ, 2021). Die Präsidentin der EZB Christine Lagarde hat entsprechend signalisiert, dass auch mit dem Auslaufen des Pandemischen Notfallkaufprogramms im Jahr 2022 kein wesentlicher Rückgang der Ankäufe von Staatsanleihen zu erwarten sei (EZB, 2021b). Sollte die Konsumentenpreisinflation weiter hoch bleiben, könnte dies den Druck erhöhen, das neue Inflationsziel deutlich höher als $2 \%$ zu setzen (oder die Inflation niedriger zu messen). ${ }^{7}$

Denn nicht nur die geldpolitische Strategie, sondern auch die Art der Inflationsmessung hat sich seit Einführung des

6 Eichenbaum et al. (2021) schätzen z. B. für Portugal, dass von März bis Mai 2020 die Konsumausgaben der 20- bis 49-Jährigen um ca. $19,2 \%$, der 50-Jährigen um ca. 23,8\%, der 60-Jährigen um ca. $27,1 \%$ und der 70 -Jährigen um ca. $34 \%$ zurückgegangen sind.

7 Roach (2021) schreibt zu den Änderungen bei der Inflationsmessung in den USA in den 1970er Jahren, dass unter anderem Energie und Nahrungsmittel aus dem Index genommen wurden, um die offiziell gemessene Inflation niedrig zu halten. „Wir wussten das damals nicht, doch wir hatten so gerade die erste Version dessen erschaffen, was heute gern als Kerninflationsrate bezeichnet wird: den bereinigten Teil des Verbraucherpreisindex, der angeblich frei ist von den schwankungsanfälligen Sonderfaktoren Lebensmittel und Energie, bei denen sich Verwerfungen auf weit entfernte Kriege und Wetterereignisse zurückführen lassen.“ 
Euro maßgeblich verändert, ${ }^{8}$ bzw. wurde an wichtigen Stellen nicht verändert. Während die EZB den HVPI als

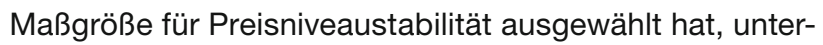
liegt die Entscheidung wie der HVPI gemessen wird dem Europäischen Rat, dem Europäischen Parlament und der Europäischen Kommission. Die Inflationsmessung ist somit Ergebnis politischer Entscheidungen. Seit Beginn der gemeinsamen Geldpolitik gab es zahlreiche neue Richtlinien, welche die Inflationsmessung verändert haben, was insbesondere bei einem fixierten Inflationspunktziel indirekt auf die Ausgestaltung der Geldpolitik der EZB wirkt. Drei Punkte sind wichtig:

Erstens werden Qualitätsverbesserungen zunehmend zum Anlass genommen, die in den Läden gemessenen Preise in der amtlichen Statistik nach unten zu korrigieren (Schnabl 2020, Schnabl und Sepp, 2020). Die Forcierung der Qualitätsanpassung bei der Preismessung hat ihren Ursprung in den USA im Boskin Report, der feststellte, dass aufgrund nicht ausreichender Qualitätsanpassungen bei der Preismessung die Inflation zu hoch gemessen würde (Boskin et al., 1996). ${ }^{9}$ Seit 1999 wurden zahlreiche EU-Richtlinien mit neuen Vorgaben zur Qualitätsanpassung verabschiedet. ${ }^{10}$ Es gibt jedoch bislang immer noch keinerlei methodische und empirische Transparenz, in welchem Umfang und nach welchen Kriterien Qualitätsanpassungen welcher Länder im Euroraum erfolgen.

Doch scheint es, dass die Qualitätsanpassungen vor allem bei Industriegütern und Wohnen erfolgt, wo Qualitätsverbesserungen dominieren dürften. Bei Dienstleistungen, bei denen oft schwindende Qualität zu beobachten ist, scheint das weit weniger der Fall. Das Statistische Bundesamt gibt keine Auskunft darüber, bei welchen Gütern konkret Qualitätsanpassungen erfolgen und wie hoch der Einfluss der Qualitätsanpassung auf die offiziell

8 Aus dieser Sicht sind Vergleiche der heutigen realen Zinsen mit den realen Zinsen der 1970er Jahre nicht belastbar, weil sich die Inflationsmessung maßgeblich verändert hat. Für die USA misst Williams (2021) eine Schatteninflationsrate, die den Index seit 1980 bzw. 1990 unverändert lässt. Dies führt zu deutlich höheren Inflationsraten für die USA als die offiziell gemessenen.

9 Die Ausweitung der Qualitätsanpassungen bei der Preismessung ging von der Annahme aus, dass ohne ausreichende Qualitätsanpassung die Inflationsraten zu hoch gemessen würden (Boskin et al., 1996). Zwar werden theoretisch auch Qualitätsverschlechterungen, z. B. wenn ein Autohersteller für einen neuen Fahrzeugtyp einen höheren Benzinverbrauch ausweist, zum Anlass genommen, die Preise herunter zu rechnen (Hagenkort und Sewald, 2021), doch dürften in der Praxis von den Herstellern hervorgehobene Qualitätsverbesserungen dominieren.

10 Konkret wurden folgende Verordnungen erlassen, wo es neue Vorgaben zur Qualitätsanpassung gab: Kommissionsverordnung 1749/96, Kommissionsverordnung 2646/98, Kommissionsverordnung 1617/1999, Ratsverordnung 2166/1999, Kommissionsverordnung 2602/2000, Kommissionsverordnung 1920/2001, Kommissionsverordnung 1334/2007, Verordnung des Europäischen Parlaments und Rats 2016/792 und Durchführungsverordnung der Kommission 2020/1148.

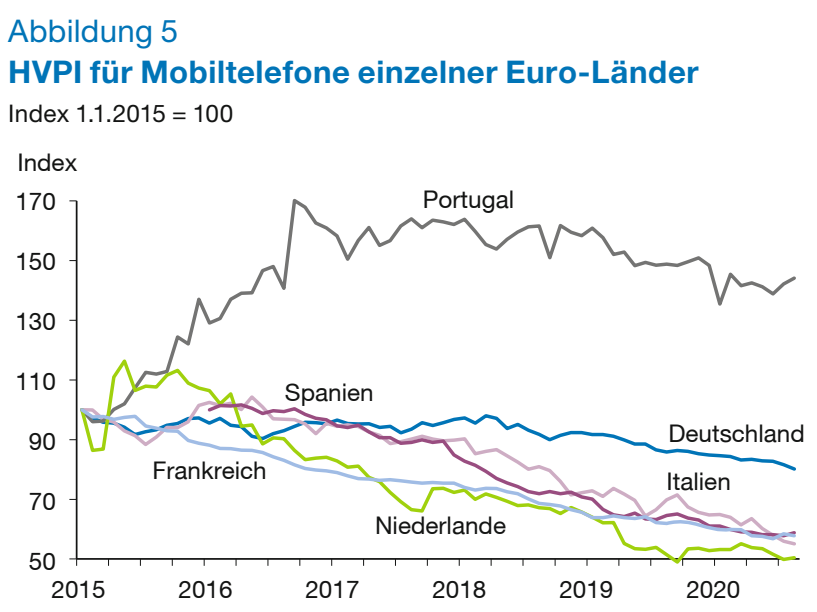

Quelle: Eurostat.

gemessene Verbraucherpreisinflation bzw. die HVPI-Rate ist (Hagenkort-Rieger und Sewald, 2021).

Zudem scheint es, dass innerhalb des Euroraums die Qualitätsanpassung von den nationalen Statistikämtern sehr unterschiedlich gehandhabt wird. Abbildung 5 zeigt die Entwicklung der Preisindizes der nationalen statistischen Behörden für Mobiltelefone. In Deutschland, Italien, Spanien, Frankreich, Niederlande und Portugal entwickeln sich diese Teilindizes sehr unterschiedlich, obwohl man davon ausgehen kann, dass in dem Gemeinsamen Markt der EU die Preisentwicklungen ähnlich sein dürften. Bei öffentlichen Gütern, wo sich z. B. in den vergangenen Monaten die Qualität deutlich verschlechtert haben dürfte (z. B. Schulbildung) erfolgt schon allein deshalb keine Qualitätsanpassung (hier durch höhere Preise in der Statistik), weil diese Güter nicht im Warenkorb vertreten sind. ${ }^{11}$

Zweitens werden Gütergruppen, deren Preise von der Geldpolitik der EZB stark beeinflusst werden, bei der Inflationsmessung im Euroraum ausgeklammert. Der Konsumentenpreisindex ist damit kein Maß für allgemeine Inflation (International Labor Organisation, 2020) wie oft in der öffentlichen Berichterstattung durch den Begriff „Inflation“ für "Konsumentenpreisinflation“ suggeriert wird. ${ }^{12}$

11 Aus dieser Sicht kann auch bei der Qualitätsanpassung zwischen einer Verzerrung durch fehlende Güter (hier öffentliche Güter) und einer von der Qualitätsanpassung ausgeschlossenen Gütern (unter Umständen Dienstleistungen) unterschieden werden.

12 „A CPI is not a measure of general inflation, as it only measures changes in the prices of consumer goods purchased by households. A CPI does not cover capital goods, such as housing, or the goods and services consumed by enterprises or the government [...]." (International Labor Organisation, 2020, 30). Der Konsumentenpreisindex wäre nur dann ein zuverlässiges $\mathrm{Maß}$ für die Inflation, wenn seine Entwicklung mit jenen Gütern, die nicht erfasst sind, eng korrelieren würde, oder diese Güter nur relativ wenig von den privaten Haushalten nachgefragt würden. 
Ausgeschlossene Güter sind insbesondere Immobilien, Aktien und öffentliche Güter. ${ }^{13}$

Obwohl die expansive Geldpolitik der EZB spätestens seit der europäischen Finanz- und Schuldenkrise zu stark steigenden Vermögenspreisen beigetragen hat (Schnabl, 2019), wurde die Frage, wie die Vermögenspreisinflation in die Preismessung mit einbezogen werden kann, vernachlässigt. ${ }^{14}$ Das gilt auch für eigengenutzte Immobilien, die bis heute im HVPI ausgeklammert bleiben (Israel und Schnabl, 2020) und deren Preise in Deutschland seit 2010 stark gestiegen sind (vgl. Abbildung 6). Eine starke Verzerrung ergibt sich dadurch, dass die Vermögenspreise von der Preismessung ausgeschlossen sind, obwohl sie nicht mit dem Konsumentenpreisindex korreliert sind. ${ }^{15}$

Drittens wird die Inflationsmessung beim HVPI in einem Abstand von zwei Jahren den veränderten Konsumgewohnheiten angepasst (Israel und Schnabl, 2020). ${ }^{16}$ Konkret werden die Gewichte von Gütern erhöht (gesenkt),

13 Grundsätzlich kann zwischen fehlenden (missing goods) und ausgeschlossenen Gütergruppen (excluded goods) unterschieden werden. Fehlende Gütergruppen sind jene Waren oder Dienstleistungen, die konzeptionell im Güterkorb enthalten sind, für die aber vorübergehend oder ab einem gewissen Zeitpunkt keine validen Preisinformationen mehr vorliegen (z. B. Saisonwaren bei Bekleidung oder bei Sortimentswechsel). Hier fehlen geeignete Preisrepräsentanten infolge von Datenverfügbarkeitsproblemen. Auch die fehlenden Daten im Zuge der erzwungenen Schließungen und die sich daraus ergebende Imputation von Daten kann unter diesem Gesichtspunkt gesehen werden (Sewald und Hagenkort-Rieger, 2021). Ausgeschlossene Gütergruppen sind Waren oder Dienstleistungen, die aus konzeptionellen Gründen von einer Aufnahme in den Warenkorb ausgeschlossen sind, die aber wichtige Informationen über die Entwicklung der Kaufkraft des Geldes enthalten, z.B. öffentliche Güter im Sinne des VGR-Verbrauchskonzepts oder Vermögensgüter, die dem zukünftigen Konsum dienen.

14 Hagenkort und Sewald $(2021,24)$ geben dafür folgende Gründe an: „Preisschwankungen von Vermögenswerten - etwa die Verteuerung von Aktien - haben keinen unmittelbaren Einfluss auf die Kaufkraft des Euro für die Lebenshaltung. Würde man dennoch die steigenden Aktienkurse im Verbraucherpreisindex berücksichtigen, wäre seine Aussagekraft für die Ziele der Wirtschafts- und Geldpolitik eingeschränkt bis unbrauchbar. Im Zuge der Anpassung von Mietzahlungen würden sich die Schwankungen der Aktienkurse dann beispielsweise über die im VPI mit hohem Wägungsanteil enthaltenen Mieten „doppelt“ in der Preisentwicklung auswirken: Steigt der VPI schneller an, werden auch indexgebundene Mieten in kürzeren Abständen angehoben, was wiederum erhöhend auf den Verbraucherpreisindex wirkt." Anmerkung: Der Anteil der Kaltmieten im Warenkorb ist im deutschen VPI 20,7\%, im für die Geldpolitik relevanten HVPI von Eurostat für Deutschland hingegen nur 10,4 \% (Mai 2021).

15 In diesem Kontext ist auch das Argument interessant, dass Zentralbanken Deflation, d.h. fallende Preise, aufgrund der negativen Konjunktureffekte der Deflation verhindern müssten. Denn wäre die Inflation im Euroraum unter Berücksichtigung der Vermögenspreise anders gemessen worden, hätte es auch keine deflationären Tendenzen „gegeben“.

16 Auch die Anpassung der Gewichte im Laspeyres-Index geht auf die Boskin-Kommission (Boskin et al., 1996) zurück, die einen Substitutions-Bias feststellte und diesen für die USA auf jährlich 0,4 Prozentpunkte schätzte. Er tritt auf, weil Konsumenten Produkte, die relativ teurer werden, durch relativ günstigere Substitute ersetzen, was nicht berücksichtigt wird, wenn der Warenkorb und die Gewichte der Produkte über die Zeit gleichbleiben (Israel und Schnabl, 2020).
Abbildung 6

HVPI, Immobilienpreisindex, Aktienpreisindex für

Deutschland

Index Q1.1999 = 100, jeweils 1. Quartal

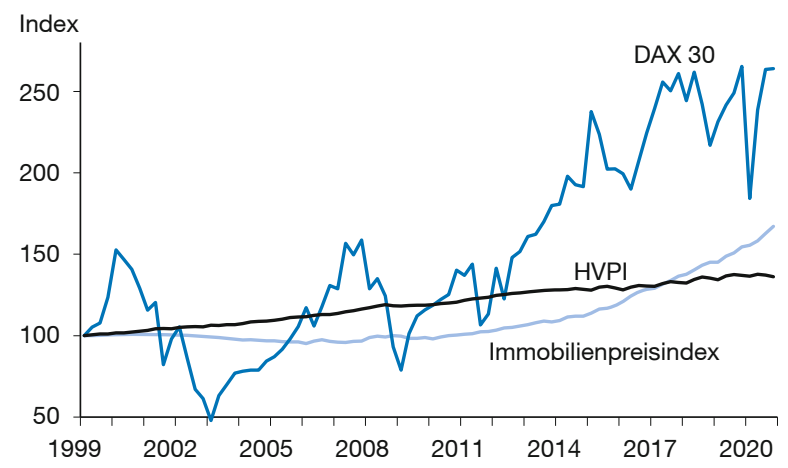

Quelle: Deutsche Bundesbank.

wenn die Konsument:innen diese Güter mehr (weniger) nachfragen. ${ }^{17}$ Damit ist folgendes Szenario denkbar: Wenn der Lebensstandard fällt - beispielsweise, weil eigengenutzte Immobilien und die Altersvorsorge deutlich teurer werden und deshalb für viele Haushalte weniger Budget für den täglichen Konsum verfügbar ist -, dann ersetzen Menschen Güter mit hohen Preissteigerungen durch Güter mit geringer Preissteigerung. Dieser Substitutionseffekt materialisiert sich in sinkender offiziell gemessener Inflation, weil das Ausgabengewicht von Gütern mit geringen Inflationsraten im HVPI erhöht wird. Geringe offiziell gemessene Inflationsraten spiegeln dann nicht zwingend eine stabile Kaufkraft, sondern unter Umständen sogar eine geringere Kaufkraft der Bürger:innen wider.

Einen besonderen Kniff bei der Gewichtung hat die europäische Statistikbehörde Eurostat im Zuge der CoronaKrise angewendet. 2020 ist aufgrund der weitreichenden Lockdown-Maßnahmen der Konsum von Dienstleistungen in Hotels und Gaststätten etc. deutlich zurückgegangen. Das hätte normalerweise dazu geführt, dass 2022 das Gewicht dieser Dienstleistungen im HVPI reduziert

17 Folgende Verordnungen mit neuen Vorgaben zur Gewichtsanpassung wurden erlassen: Ratsverordnung 2494/95, Kommissionsverordnung 1749/96, Kommissionsverordnung 2214/96, Kommissionsverordnung 2454/97, Ratsverordnung 1687/98, Ratsverordnung 1688/98, Kommissionsverordnung 2646/98, Kommissionsverordnung 1617/1999, Ratsverordnung 2166/1999, Kommissionsverordnung 1920/2001, Kommissionsverordnung 1921/2001, Kommissionsverordnung 1708/2005, Kommissionsverordnung 1334/2007, Kommissionsverordnung 330/2009, Kommissionsverordnung 1114/2010, Kommissionsverordnung 93/2013, Kommissionsverordnung 113/2013, Verordnung des Europäischen Parlaments und Rats 2016/792 und Durchführungsverordnung der Kommission 2020/1148. 
wird. Nun wurde überraschend diese Anpassung auf 2021 vorgezogen (Destatis, 2021). Das dürfte den Effekt haben, dass 2021 die offiziell gemessenen Inflationsraten aufgrund eines reduzierten Gewichts für Dienstleistungen niedriger ausgewiesen werden. Denn es zeichnet sich ab, dass mit Öffnung der Dienstleistungsbetriebe die Preise deutlich steigen werden.

Die 2021 anstehende Überprüfung der geldpolitischen Strategie der EZB sollte zum Anlass genommen werden, diese konzeptionellen Schwachpunkte ernsthaft anzugehen. Das könnte beispielsweise dadurch erreicht werden, dass man zur ursprünglichen Obergrenze bei der Konsumentenpreisinflation von $2 \%$ zurückkehrt. Wenn man den Referenzwert für das Geldmengenwachstum wieder aufnehmen und ernst nehmen würde, könnte man den Einfluss monetärer Größen auf die Vermögenspreise stärker berücksichtigen. Es ist genau zu überprüfen, inwieweit die EZB diesen Weg einschlagen wird oder ob sie sich weiter von einer nachhaltigen Geldpolitik mit dem klaren Primat der Preisstabilität entfernt.

\section{Literatur}

Boskin, M., E. Dulberger, R. Gordon, Z. Griliches und D. Jorgenson (1996), Toward a More Accurate Measure of the Cost of Living, Final Report to the U.S. Senate Finance Committee.

Constancio, V. (2018), Past and future of the ECB monetary policy, https://www.ecb.europa.eu/press/key/date/2018/html/ecb.sp180504. en.html (11. Juni 2021).

Destatis (2021), Ableitung des HVPI-Wägungsschemas für das Jahr 2021, https://www.destatis.de/DE/Themen/Wirtschaft/Preise/Verbraucherpreisindex/Methoden/Downloads/hvpi-gewichtung.pdf? blob=publicationFile (11. Juni 2021).

Draghi, M. (2012), Verbatim of the remarks made by Mario Draghi, https:// www.ecb.europa.eu/press/key/date/2012/html/sp120726.en.html (11. Juni 2021).

Europäische Kommission (2021), Qualitative and Quantitative Inflation perceptions, https://ec.europa.eu/info/business-economy-euro/ indicators-statistics/economic-databases/business-and-consumersurveys/download-business-and-consumer-survey-data/time-series_en (11. Juni 2021).

Europäische Zentralbank (1999), EZB-Monatsbericht Januar 1999.

Europäische Zentralbank (2003), The ECB's monetary policy strategy, https://www.ecb.europa.eu/press/pr/date/2003/html/pr030508_2. en.html (11. Juni 2021).

Eichenbaum, M., M. Godinho de Matos, F. Lima, S. Rebelo und M. Trabandt (2020), How Do People Respond to Small Probability Events with Large, Negative Consequences?, NBER Working Paper, 27988.

FAZ (2021), Bundesbank hält 4 Prozent Inflation für möglich, https://www. faz.net/aktuell/finanzen/bundesbank-haelt-4-prozent-inflation-fuermoeglich-17354245.html (11. Juni 2021).

Hagenkort-Rieger, S. und N. Sewald (2021), Theoretische und Praktische Ansätze der Inflationsmessung in Zeiten der Corona-Pandemie, WISTA, 1, 2021, 19-33.

International Labor Organisation (2020), Consumer Price Index Manual Concepts and Methods 2020, International Labor Organisation.

Israel, K.-F. und G. Schnabl (2020), Alternative Measures of Price Inflation and the Perception of Real Income in Germany, CESifo Working Paper, 8583.

Lagarde, C. (2020), The monetary policy strategy review: some preliminary considerations, https://www.ecb.europa.eu/press/key/date/2020/ html/ecb.sp200930 169abb1202.en.html (11. Juni 2021).

Linz, S. und G. Eckert (2002), Zur Einführung hedonischer Methoden in die Preisstatistik, Statistisches Bundesamt - Wirtschaft Und Statistik, 10, 857-863.

ntv (2021), EZB-Direktorin im ntv-Interview, https://www.n-tv.de/ wirtschaft/Schnabel-sieht-kraeftigen-Anstieg-der-Inflation-article22546945.html (11. Juni 2021).

Roach, S. (2021), Der Geist von Arthur Burns, Finanz und Wirtschaft, 2. Juni.

Schnabl, Gunther (2020), Die Inflationsmessung erzeugt die Illusion der stabilen Kaufkraft, Wirtschaftsdienst, 100(11), 838-841, https://www. wirtschaftsdienst.eu/inhalt/jahr/2020/heft/11/beitrag/die-inflationsmessung-erzeugt-die-illusion-der-stabilen-kaufkraft.html (8. Juli 2021).

Schnabl, G. und T. Sepp (2020), Die Qualitätsanpassung in der Verbraucherpreismessung schafft die Illusion niedriger Inflation, Wirtschaftliche Freiheit, 19. Juni.

Sonnenberg, N. und G. Schnabl (2020), (T)LTRO-Tracker, Mimeo, https://papers.ssrn.com/sol3/papers.cfm?abstract_id=3612642 (8. Juli 2021).

Stark, J., T. Mayer und G. Schnabl (2020), Geldpolitik ist eine Kunst, Die Welt, 20. Juni, 2.

Williams, J. (2021), Shadow Government Statistics, http://www.shadowstats.com/alternate_data/inflation-charts (11. Juni 2021).

Title: Inflation Targeting and Inflation Measurement in the Euro Area in Transition

Abstract: Many people in the euro area increasingly feel that the general loss of purchasing power of money is much higher than the officially measured inflation rates. For example, according to survey results, perceived inflation in the first quarter of 2021 in the euro area was $4.5 \%$, while the officially measured inflation rate was $1 \%$. This could be due to the fact that price increases are subjectively perceived to be stronger than price decreases. But it could also be due to how price stability is measured and how the European Central Bank's implementation of the price stability objective has changed.

JEL Classification: E31, E50 